\title{
Adherens junctional associated protein-1: A novel 1p36 tumor suppressor candidate in gliomas (Review)
}

\author{
LIANG ZENG ${ }^{1,2}$, BRIAN E. FEE ${ }^{2,4}$, MIRIAM V. RIVAS ${ }^{2,4}$, JAMES LIN $^{2}$ and DAVID CORY ADAMSON ${ }^{2-4}$ \\ ${ }^{1}$ Department of Neurosurgery, Tongji Hospital, Huazhong University of Science and \\ Technology, Wuhan, P.R. China; Departments of ${ }^{2}$ Surgery (Neurosurgery) and ${ }^{3}$ Neurobiology, \\ Duke University Medical Center; ${ }^{4}$ Durham VA Medical Center, Durham, NC, USA
}

Received February 20, 2014; Accepted April 14, 2014

DOI: $10.3892 /$ ijo.2014.2425

\begin{abstract}
In a broad range of human cancers 1p36 has been a mutational hotspot which strongly suggests that the loss of tumor suppressor activity maps to this genomic region during tumorigenesis. Adherens junctional associated protein-1 (AJAP1; also known as Shrew1) was initially discovered as a novel transmembrane protein of adherent junctions in epithelial cells. Gene profiling showed AJAP1 on 1p36 is frequently lost or epigenetically silenced. AJAP1 may affect cell motility, migration, invasion and proliferation by unclear mechanisms. AJAP1 may be translocated to the nucleus, via its interaction with $\beta$-catenin complexes, where it can regulate gene transcription, then possibly have a potent impact on cell cycling and apoptosis. Significantly, loss of AJAP1 expression predicts poor clinical outcome of patients with malignant gliomas such as GBM and it may serve as a promising tumor suppressor-related target. In this review, we summarize and discuss current knowledge that may identify AJAP1 as a tumor suppressor in gliomas.
\end{abstract}

\section{Contents}

1. Introduction

2. Genetic and epigenetic change of AJAP1

3. Continuing studies on AJAP1

4. Relationship between AJAP1 and tumors

5. Closing remarks

\section{Introduction}

In a broad range of human cancers $1 \mathrm{p} 36$ has been a mutational hotspot, including those of nervous, secretory, reproductive and

Correspondence to: Professor David Cory Adamson, Department of Surgery, Duke University Medical Center, DUMC Box 2624, 421-MSRB Bldg., Research Drive, Durham, NC 27710, USA

E-mail: cory.adamson@duke.edu

Key words: adherens junctions-associated protein, tumor suppressor, glioma internal origin (Fig. 1) (1-11). These studies strongly suggest that the loss of tumor suppressor activity maps to this genomic region during tumorigenesis. The large 1p36 deletions led others to propose that more than one tumor suppressor-related gene may reside in this region $(4,12)$. Although the quest for the 1 p36 tumor suppressor has led to some exciting candidates, such as CHD5, CAMTA1, KIF1B, CASZ1 and miR-34a which have tumor suppressor-like capabilities in specific tumor contexts (13-23), none can provide convincing evidence that their encoded products offer protection from cancer. Hence, the search for the 1p36 tumor suppressor continues.

Adherens junctional associated protein-1 (AJAP1; also known as Shrew1) was initially discovered as a novel transmembrane protein of adherent junctions in epithelial cells (24). Recent investigations have suggested the AJAP1 gene as a promising tumor suppressor candidate gene located at $1 \mathrm{p} 36.32$ $(13,24)$.

\section{Genetic and epigenetic change of AJAP1}

Significant data supports the role of AJAP1 loss in various tumors of the central nervous system. A single region within 1 p36.3 consistently deleted in $25 \%$ of neuroblastomas was defined by White and colleagues; AJAP1 was 1 of 6 predicted genes in this deleted region (25). Neuroblastomas are neuroendocrine tumors arising from neural crest derivatives of the sympathetic nervous system (26). Fujita et al identified 23 genes, while mapping the smallest region of a consistently deleted segment of 1p36 in neuroblastomas; AJAP1 was included (27). By analyzing 430 primary neuroblastomas, Okawa et al mapped the smallest region of deletion to a $2-\mathrm{Mb}$ region of 1p36 using microsatellite and single nucleotide polymorphisms and identified 23 genes in this region; including AJAP1 (13). Milde et al found the loss of AJAP1 in a progressively metastasizing ependymoma (28). Ependymomas are tumors that arise from the specialized ependyma which primarily lines the ventricular surface of the central nervous system (29). Dong et al reported two contiguous minimally deleted regions on chromosome 1p36.31-p36.32 in oligodendroglial tumors, one of which contained AJAP1 (30). Oligodendrogliomas are believed to originate from the myelin-producing oligodendrocytes of the brain (31). McDonald et al evaluated 177 oligodendroglial tumor samples and found a consensus region 


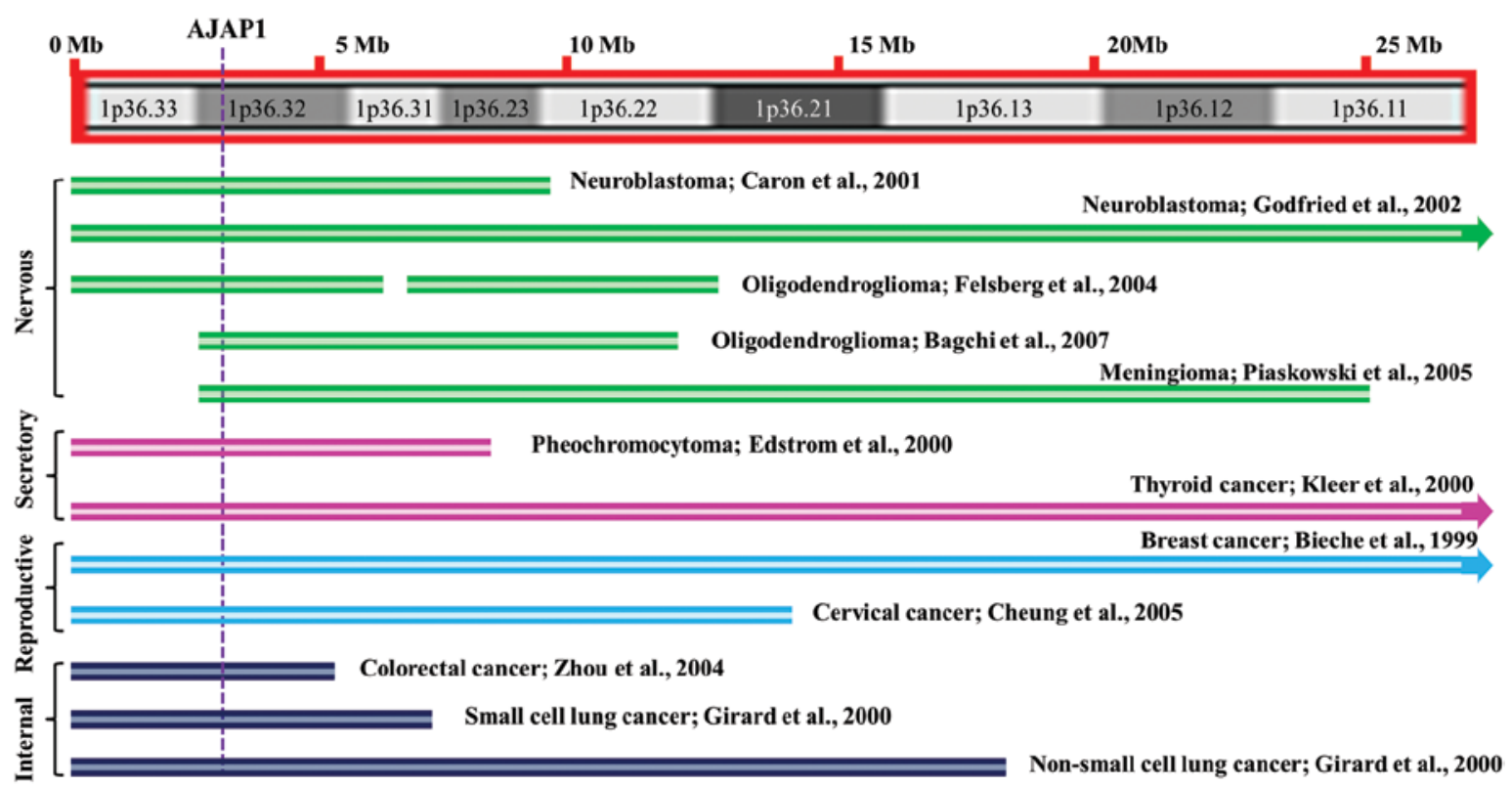

Figure $1.1 \mathrm{p} 36$ is deleted in a variety of human cancers. $1 \mathrm{p} 36$ region is shown and depicted by a gray rectangle. A subset of deletions that have been reported in a variety of nervous (green), secretory (pink), reproductive (light blue) and internal (dark blue) origin malignancies is shown to scale. All of these deletions encompass AJAP1 (dotted line).

of deletion of approximately $630 \mathrm{~kb}$. This region encompasses only one single gene - AJAP1 (32). Historically, brain gliomas have been described as likely arising from unique glial cells; however, more recent studies of genomic changes in various glioma types suggest there may be common cells of origin with common genomic changes, such as loss of AJAP1 expression.

For any tumor-suppressor activity of AJAP1 to be inhibited in a tumor, both copies of the gene must be abrogated, either by deletion or a reduction in expression. Since AJAP1 expression is found to be low in the majority of glioma tumors and cell lines $(13,24,28,32,33)$, the remaining allele of AJAP1 has been sequenced to identify mutations, revealing none in the coding region $(13,32,33)$. As shown for some tumor suppressors, decreased gene dosage predisposes cells to tumorigenesis. Additionally, as the expression of AJAP1 is often reduced or absent irrespective of a deletion at that locus, epigenetic silencing has been investigated. Bioinformatic analysis revealed the presence of numerous $\mathrm{CpG}$ islands in the promoter of AJAP1, the favored site for methylation-regulation of gene expression (33). Methylation analysis of the AJAP1 promoter identified hypermethylation in $21 \%$ of oligodendrogliomas, and the degree of methylation inversely correlated with AJAP1 expression. The AJAP1 promoter was also highly methylated in a wide spectrum of cell lines (24).

Using multiple high-resolution genomic screening methods, we found AJAP1 frequently lost or epigenetically silenced in many glioblastomas (GBM) (33). Glioblastomas which arise from astrocytes are the most common and most aggressive malignant primary brain glioma in humans which can be found throughout the brain or spinal cord (34). Expression studies revealed that loss of AJAP1 gene expression is more commonly due to epigenetic silencing of the gene than gene deletion. Expression was reduced or absent in 86-92\% of primary GBM tumors (those with no history of prior lower grade tumors) and all glioma cell lines, whereas the gene was deleted in only

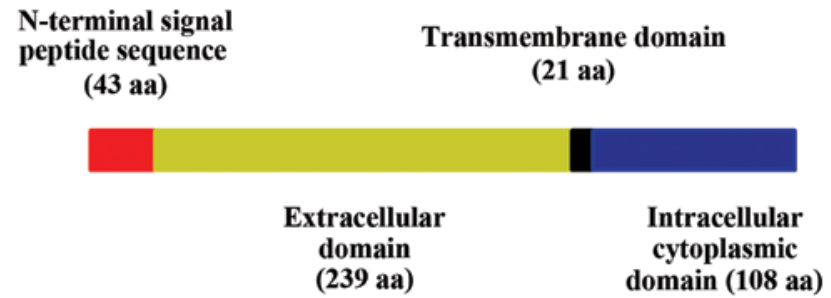

Figure 2. The four structural domains of the 411 amino acid residue AJAP1 protein.

$16 \%$ of the tumor samples. Using mutational and methylation analyses, we showed that AJAP1 expression was not due to mutation but epigenetically silenced with promoter methylation in many cases (33).

\section{Continuing studies on AJAP1}

AJAP1 was initially identified by Bharti et al in epithelial cells of the uterus as a candidate gene increased in invasive endometriosis (35). Confocal microscopic analysis of transiently-expressed AJAP1 revealed its localization at the baso-lateral part of the cell membrane, where it co-localized with endogenous E-cadherin, a protein also present at the baso-lateral part of the membrane in polarized Madin-Darby canine kidney (MDCK) epithelial cells $(36,37)$. It has been demonstrated that adhesion is mediated by the cytoplasmic domain of E-cadherin linking to the actin cytoskeleton (38) via associated proteins such as $\alpha$ - and $\beta$-catenin (39). Direct interaction between $\beta$-catenin and AJAP1 in an in vitro pull-down assay suggested that AJAP1 might be directly linked to the E-cadherin via $\beta$-catenin. It can be found at the baso-lateral part of the plasma membrane where it co-localizes with, and apparently integrates into E-cadherinmediated adherens junctions (40). 


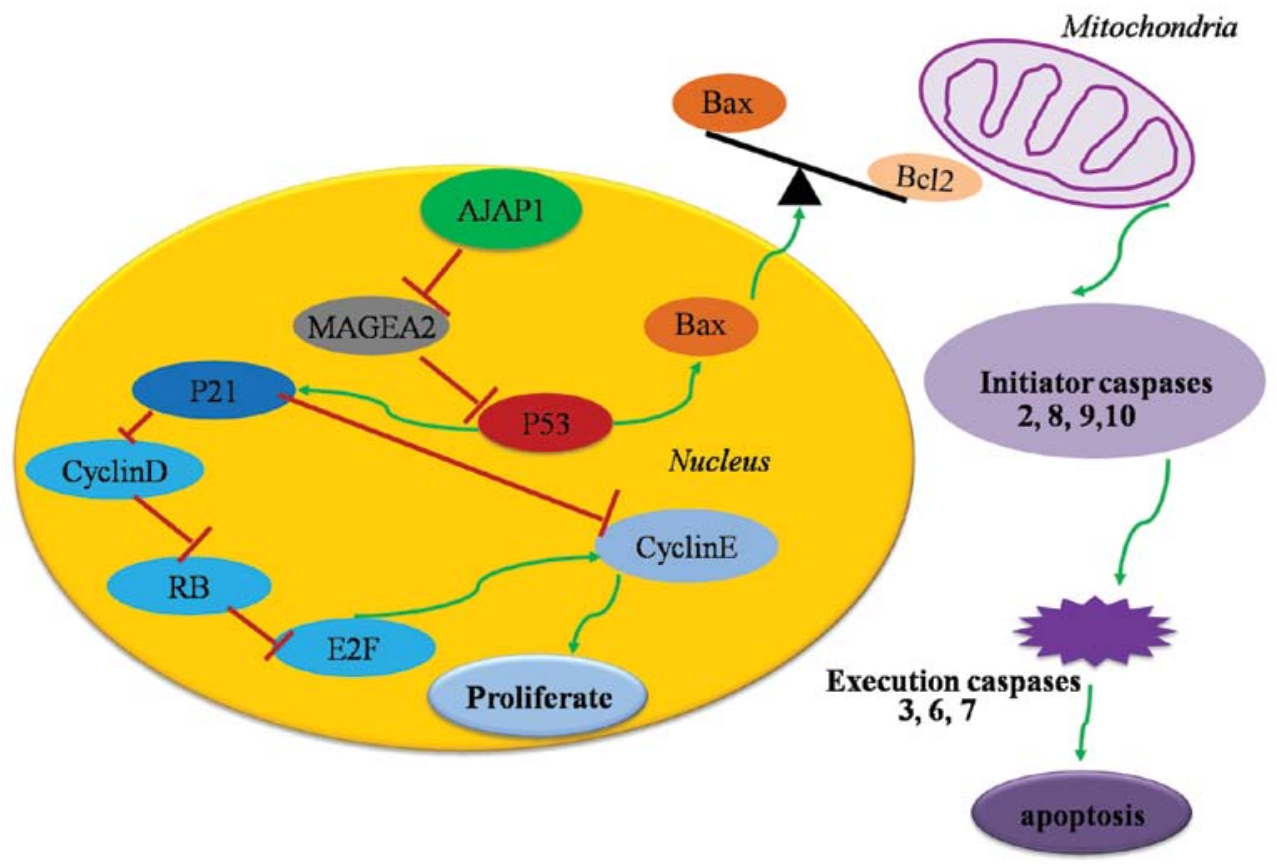

Figure 3. AJAP1 may be translocated to the nucleus, where it can regulate MAGEA2 gene transcription, then possibly have a potent impact on cell proliferation and apoptosis through P53 pathway and downstream compartment to regulate Bax/Bcl-2 ratios and caspase-3/7 activity, finally inducing mitochondria-related apoptosis.
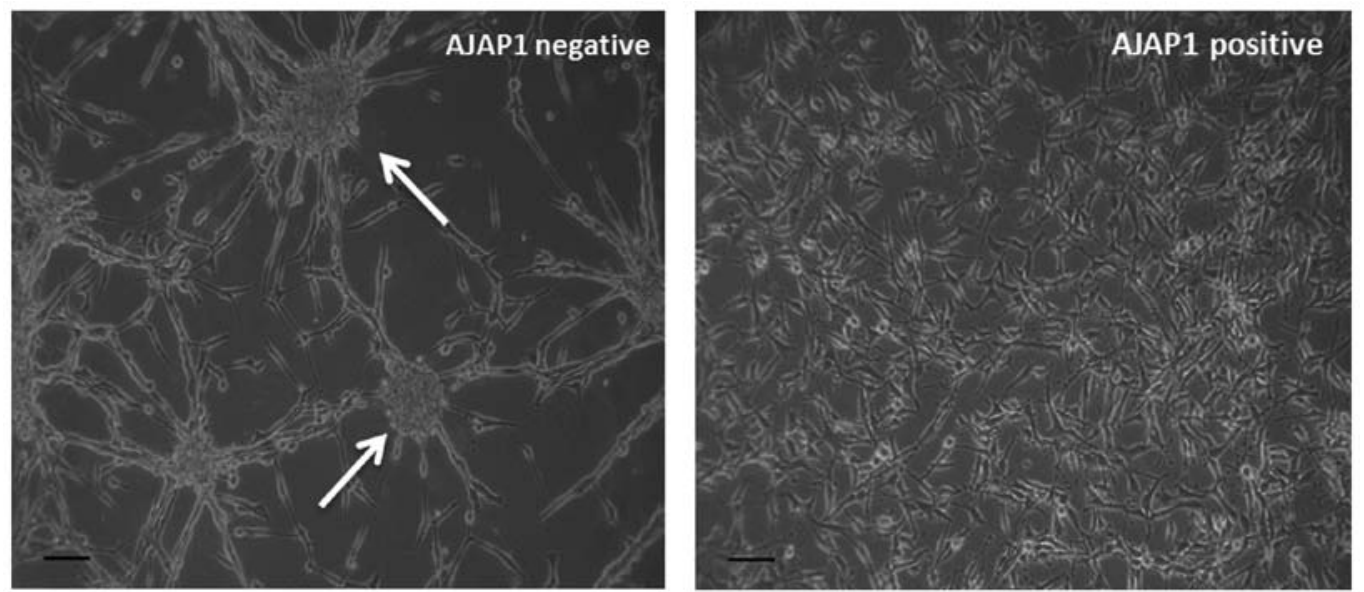

Figure 4. U87MG cells grown in culture form tumorspheres (white arrows) in the absence of AJAP1 after $72 \mathrm{~h}$. However, cells transfected with AJAP1 do not. Photos were taken at $\mathrm{x} 40$ with a light microscope. Scale bar represents 100 microns.

In polarized MCF7 mammary epithelial cells and MDCK cells, AJAP1 is a transmembrane protein which co-localizes with endogenous E-cadherin (35). Additionally, CD147 was shown to interact with AJAP1 and increase cell invasion (41). However, AJAP1 does not appear to interact with the $\mathrm{N}$-cadherin complex in non-polarized epithelial cells (35). These findings suggest a potential role for AJAP1 in cell-cell and cell-extracellular matrix interactions that could be involved in cell motility, migration and invasion.

Apparently conflicting results are associated with AJAP1 expression. In HeLa cells (derived from a cervical tumor), loss of AJAP1 expression results in decreased invasiveness (41); however, its overexpression in glioma cells also results in decreased invasiveness, McDonald et al found in U251 cells (glioma cells) that AJAP1 overexpression decreased cell adhesion on extracellular matrix components and decreased migration in wound healing assays (32). These studies demonstrate that the effect of AJAP1 may depend on the specific cell type and its environment.

AJAP1 is an integral membrane protein of 411-amino acid residues (35) and is comprised of a cleavable N-terminal signal peptide (residues 1-43), an extracellular domain (residues 44-282), a transmembrane domain (residues 283-303) and an intracellular cytoplasmic domain (residues 304-411) (Fig. 2) (42). The relatively long signal peptide also has a role in the cellular location of AJAP1 $(43,44)$. The signal peptide of AJAP1 conforms to the NtraC model describing the organization of long signal peptides, i.e., an N-terminal subdomain, 
a $\beta$-turn-rich transition area, and a C-terminal subdomain. The C-domain, either by itself or with the rest of the signal peptide, allows the targeting of nascent AJAP1 to the rough endoplasmic reticulum and subsequent transfer to the plasma membrane. The N-domain alone directs AJAP1 primarily to mitochondria. This cryptic mitochondrial targeting signal has been speculated to be active only under certain physiological conditions, e.g., apoptosis $(43,44)$. Further analysis of the AJAP1 protein sequence revealed a putative nuclear localization signal in the predicted extracellular domain and putative glycosylation signals in the cytoplasmic domain (35).

Direct interaction between $\beta$-catenin and AJAP1 in an in vitro pull-down assay suggested that AJAP1 might be linked to the E-cadherin-mediated junctional complexes via $\beta$-catenin (35). $\beta$-catenin contains armadillo repeats and is able to bind to other proteins such as transcription factors (45). The ability of $\beta$-catenin to bind to other proteins is regulated by tyrosine kinases (46) and serine kinases such as GSK-3 (47). Various signals such as the Wnt signaling pathway can inhibit GSK-3mediated phosphorylation of $\beta$-catenin, allowing $\beta$-catenin to translocate to the cell nucleus, interact with transcription factors, and regulate gene transcription (48). AJAP1 may be translocated to the nucleus, via its interaction with $\beta$-catenin complexes, where it can regulate gene transcription, therefore possibly having a potent impact on cell cycling and apoptosis (Fig. 3). One of our studies suggested a regulatory effect of AJAP1 on Bax/Bcl-2 ratios with increased caspase-3/7 activity in the presence of AJAP1, which indicated that AJAP1 could, at least in part, induce the mitochondria-related apoptosis pathways (49).

\section{Relationship between AJAP1 and tumors}

Cogdell and colleagues suggested overexpression of AJAP1 could suppress cell invasion and migration in GBM cell lines (24). We identified loss of AJAP1 expression as being associated with increased glioma cell proliferation and migration (33).

Interaction with $\beta$-catenin, a critical member of the Wnt signaling pathway, also suggests a possible role of AJAP1 in glioma stem cell (GSC) modulation. We observed that in the presence of AJAP1, U87MG cells (a GBM cell line) do not form tumorspheres while in the absence of AJAP1, get tumorspheres (Fig. 4). When probed for CD133, a marker of GSCs, we also noticed a decrease in its expression in AJAP1 transfected cells (data not shown) indicating either a loss of stem-like properties of the cell or decrease in the GSC subpopulation.

The group of Radlwimmer (50) performed comparative genomic hybridization and microarray analysis of spheroid cultures from GBM patients, where AJAP1 as well as EMP3 and PDPN were first described as novel candidate genes that likely play a role in GBM pathogenesis and biomarkers associated with GBM outcome. By analyzing the National Cancer Institute's Rembrandt dataset, which contains 343 glioma samples, McDonald and colleagues (32) indicated that low AJAP1 gene expression was associated with decreased survival. Both genetic (gene deletion) and epigenetic alterations (promoter methylation) are likely mechanisms that inactivate the putative tumor suppressor AJAP1 in gliomas, which contributes to poor prognosis (24). We and others also demonstrated that the AJAP1 promoter was highly methyl- ated in a wide spectrum of glioma cell lines, and the loss of expression was associated with poorer survival in gliomas patients (33). We recently showed that at an early stage of gliomagenesis AJAP1 is dysregulated and may suppress invasion through cytoskeleton reorganization associated with actin and microtubules associations (51). Similar result was found in a cohort study of cervical cancer (8). Restoration of AJAP1 gene expression by transfection or demethylation agents results in decreased tumor cell migration in GBM cell lines. These investigations show the significant loss of expression of AJAP1 in GBM and provide evidence of its role in the highly migratory characteristic of these tumors (33).

\section{Closing remarks}

In summary, gene profiling showed AJAP1 on 1p36 is frequently lost or epigenetically silenced in various kinds of human cancers. AJAP1 may affect cell motility, migration, invasion and proliferation by unclear mechanisms in different cell types. In particular, loss of AJAP1 expression predicts poor clinical outcome of patients with malignant gliomas such as GBM and it may serve as a promising tumor suppressor-related target. Although AJAP1 does not conform to the classical definition of a tumor suppressor, accumulating evidence from studies on other human cancers may identify AJAP1 as a tumor suppressor via the alternate dosage hypothesis.

\section{References}

1. Caron H, Spieker N, Godfried M, et al: Chromosome bands 1p35-36 contain two distinct neuroblastoma tumor suppressor loci, one of which is imprinted. Genes Chromosomes Cancer 30: 168-174, 2001.

2. Godfried MB, Veenstra M, Valent A, et al: Lack of interstitial chromosome 1p deletions in clinically-detected neuroblastoma. Eur J Cancer 38: 1513-1519, 2002.

3. Felsberg J, Erkwoh A, Sabel MC, et al: Oligodendroglial tumors: refinement of candidate regions on chromosome arm $1 \mathrm{p}$ and correlation of $1 \mathrm{p} / 19 \mathrm{q}$ status with survival. Brain Pathol 14: 121-130, 2004.

4. Bagchi A, Papazoglu C, Wu Y, et al: CHD5 is a tumor suppressor at human 1p36. Cell 128: 459-475, 2007.

5. Piaskowski S, Rieske P, Szybka M, et al: GADD45A and EPB41 as tumor suppressor genes in meningioma pathogenesis. Cancer Genet Cytogenet 162: 63-67, 2005.

6. Edström Elder E, Nord B, Carling T, et al: Loss of heterozygosity on the short arm of chromosome 1 in pheochromocytoma and abdominal paraganglioma. World J Surg 26: 965-971, 2002.

7. Bieche I, Khodja A and Lidereau R: Deletion mapping of chromosomal region 1p32-pter in primary breast cancer. Genes Chromosomes Cancer 24: 255-263, 1999.

8. Cheung TH, Lo KW, Yim SF, et al: Clinicopathologic significance of loss of heterozygosity on chromosome 1 in cervical cancer. Gynecol Oncol 96: 510-515, 2005.

9. Zhou CZ, Qiu GQ, Zhang F, He L and Peng ZH: Loss of heterozygosity on chromosome 1 in sporadic colorectal carcinoma. World J Gastroenterol 10: 1431-1435, 2004.

10. Girard L, Zochbauer-Muller S, Virmani AK, Gazdar AF and Minna JD: Genome-wide allelotyping of lung cancer identifies new regions of allelic loss, differences between small cell lung cancer and non-small cell lung cancer, and loci clustering. Cancer Res 60: 4894-4906, 2000.

11. Kleer CG, Bryant BR, Giordano TJ, Sobel M and Merino MJ: Genetic changes in chromosomes $1 \mathrm{p}$ and $17 \mathrm{p}$ in thyroid cancer progression. Endocr Pathol 11: 137-143, 2000.

12. Schlisio S, Kenchappa RS, Vredeveld LC, et al: The kinesin KIF1beta acts downstream from EglN3 to induce apoptosis and is a potential 1p36 tumor suppressor. Genes Dev 22: 884-893, 2008.

13. Okawa ER, Gotoh T, Manne J, et al: Expression and sequence analysis of candidates for the $1 \mathrm{p} 36.31$ tumor suppressor gene deleted in neuroblastomas. Oncogene 27: 803-810, 2008. 
14. Robbins CM, Tembe WA, Baker A, et al: Copy number and targeted mutational analysis reveals novel somatic events in metastatic prostate tumors. Genome Res 21: 47-55, 2011.

15. Sjoblom T, Jones S, Wood LD, et al: The consensus coding sequences of human breast and colorectal cancers. Science 314 268-274, 2006.

16. Ichimura K, Vogazianou AP, Liu L, et al: $1 \mathrm{p} 36$ is a preferential target of chromosome 1 deletions in astrocytic tumours and homozygously deleted in a subset of glioblastomas. Oncogene 27: 2097-2108, 2008.

17. Tanas MR, Sboner A, Oliveira AM, et al: Identification of a disease-defining gene fusion in epithelioid hemangioendothelioma. Sci Transl Med 3: 92-98, 2011.

18. Henrich KO, Fischer M, Mertens D, et al: Reduced expression of CAMTA1 correlates with adverse outcome in neuroblastoma patients. Clin Cancer Res 12: 131-138, 2006.

19. Zhang H, Zhai Y, Hu Z, et al: Genome-wide association study identifies 1 p36.22 as a new susceptibility locus for hepatocellular carcinoma in chronic hepatitis B virus carriers. Nat Genet 42 : 755-758, 2010.

20. Caren H, Fransson S, Ejeskar K, Kogner P and Martinsson T: Genetic and epigenetic changes in the common 1p36 deletion in neuroblastoma tumours. Br J Cancer 97: 1416-1424, 2007.

21. Liu Z, Yang X, Li Z, et al: CASZ1, a candidate tumor-suppressor gene, suppresses neuroblastoma tumor growth through reprogramming gene expression. Cell Death Differ 18: 1174-1183, 2011.

22. He L, He X, Lim LP, et al: A microRNA component of the p53 tumour suppressor network. Nature 447: 1130-1134, 2007.

23. Guessous F, Zhang Y, Kofman A, et al: microRNA-34a is tumor suppressive in brain tumors and glioma stem cells. Cell Cycle 9: 1031-1036, 2010

24. Cogdell D, Chung W, Liu Y, et al: Tumor-associated methylation of the putative tumor suppressor AJAP1 gene and association between decreased AJAP1 expression and shorter survival in patients with glioma. Chin J Cancer 30: 247-253, 2011.

25. White PS, Thompson PM, Gotoh T, et al: Definition and characterization of a region of $1 \mathrm{p} 36.3$ consistently deleted in neuroblastoma. Oncogene 24: 2684-2694, 2005.

26. Cheung NK and Dyer MA: Neuroblastoma: developmental biology, cancer genomics and immunotherapy. Cancer 13: 397-411, 2013.

27. Fujita T, Igarashi J, Okawa ER, et al: CHD5, a tumor suppressor gene deleted from 1p36.31 in neuroblastomas. J Natl Cancer Inst 100: $940-949,2008$

28. Milde T, Pfister S, Korshunov A, et al: Stepwise accumulation of distinct genomic aberrations in a patient with progressively metastasizing ependymoma. Genes Chromosomes Cancer 48 229-238, 2009

29. Nagasawa DT, Trang A, Choy W, et al: Genetic expression profiles of adult and pediatric ependymomas: molecular pathways, prognostic indicators, and therapeutic targets. Clin Neurol Neurosurg 115: 388-399, 2013.

30. Dong Z, Pang JS, Ng MH, Poon WS, Zhou L and Ng HK: Identification of two contiguous minimally deleted regions on chromosome 1p36.31-p36.32 in oligodendroglial tumours. Br J Cancer 91: 1105-1111, 2004.

31. Alentorn A, Sanson M and Idbaih A: Oligodendrogliomas: new insights from the genetics and perspectives. Curr Opin Oncol 24: 687-693, 2012

32. McDonald JM, Dunlap S, Cogdell D, et al: The SHREW1 gene frequently deleted in oligodendrogliomas, functions to inhibit cel adhesion and migration. Cancer Biol Ther 5: 300-304, 2006.

33. Lin N, Di C, Bortoff K, et al: Deletion or epigenetic silencing of AJAP1 on 1p36 in glioblastoma. Mol Cancer Res 10: 208-217, 2012.
34. Ohgaki $\mathrm{H}$ and Kleihues P: The definition of primary and secondary glioblastoma. Clin Cancer Res 19: 764-772, 2013.

35. Bharti S, Handrow-Metzmacher H, Zickenheiner S, Zeitvogel A, Baumann R and Starzinski-Powitz A: Novel membrane protein shrew-1 targets to cadherin-mediated junctions in polarized epithelial cells. Mol Biol Cell 15: 397-406, 2004.

36. Le Bivic A, Sambuy Y, Mostov K and Rodriguez-Boulan E: Vectorial targeting of an endogenous apical membrane sialoglycoprotein and uvomorulin in MDCK cells. J Cell Biol 110: 1533-1539, 1990.

37. Shore EM and Nelson WJ: Biosynthesis of the cell adhesion molecule uvomorulin (E-cadherin) in Madin-Darby canine kidney epithelial cells. J Biol Chem 266: 19672-19680, 1991.

38. Sako Y, Nagafuchi A, Tsukita S, Takeichi M and Kusumi A: Cytoplasmic regulation of the movement of E-cadherin on the free cell surface as studied by optical tweezers and single particle tracking: corralling and tethering by the membrane skeleton. J Cell Biol 140: 1227-1240, 1998

39. Aberle H, Butz S, Stappert J, Weissig H, Kemler R and Hoschuetzky H: Assembly of the cadherin-catenin complex in vitro with recombinant proteins. J Cell Sci 107: 3655-3663, 1994.

40. Jakob V, Schreiner A, Tikkanen R and Starzinski-Powitz A Targeting of transmembrane protein shrew-1 to adherens junctions is controlled by cytoplasmic sorting motifs. Mol Biol Cell 17: 3397-3408, 2006

41. Schreiner A, Ruonala M, Jakob V, et al: Junction protein shrew-1 influences cell invasion and interacts with invasion-promoting protein CD147. Mol Biol Cell 18: 1272-1281, 2007.

42. Resch E, Quaiser S, Quaiser T, Schneider G, Starzinski-Powitz A and Schreiner A: Synergism of shrew-1's signal peptide and transmembrane segment required for plasma membrane localization. Traffic 9: 1344-1353, 2008.

43. Hiss JA, Resch E, Schreiner A, Meissner M, Starzinski-Powitz A and Schneider G: Domain organization of long signal peptides of single-pass integral membrane proteins reveals multiple functional capacity. PloS One 3: e2767, 2008.

44. Resch E, Hiss JA, Schreiner A, Schneider G and StarzinskiPowitz A: Long signal peptides of RGMa and DCBLD2 are dissectible into subdomains according to the NtraC model. Mol Biosyst 7: 942-951, 2011.

45. Gottardi CJ and Peifer M: Terminal regions of beta-catenin come into view. Structure 16: 336-338, 2008.

46. Lilien $\mathrm{J}$ and Balsamo $\mathrm{J}$ : The regulation of cadherin-mediated adhesion by tyrosine phosphorylation/dephosphorylation of betacatenin. Curr Opin Cell Biol 17: 459-465, 2005.

47. Castellone MD, Teramoto H, Williams BO, Druey KM and Gutkind JS: Prostaglandin E2 promotes colon cancer cell growth through a Gs-axin-beta-catenin signaling axis. Science 310: 1504-1510, 2005.

48. Liu X, Rubin JS and Kimmel AR: Rapid, Wnt-induced changes in GSK3beta associations that regulate beta-catenin stabilization are mediated by Galpha proteins. Curr Biol 15: 1989-1997, 2005.

49. Zeng L, Kang C, Di C, et al: The adherens junction-associated protein 1 is a negative transcriptional regulator of MAGEA2, which potentiates temozolomide-induced apoptosis in GBM. Int J Oncol 44: 1243-1251, 2014

50. Ernst A, Hofmann S, Ahmadi R, et al: Genomic and expression profiling of glioblastoma stem cell-like spheroid cultures identifies novel tumor-relevant genes associated with survival. Clin Cancer Res 15: 6541-6550, 2009.

51. Han L, Zhang KL, Zhang JX, et al: AJAP1 is dysregulated at an early stage of gliomagenesis and suppresses invasion through cytoskeleton re-organization. CNS Neurosci Ther 20: 429-437, 2014. 